\title{
Crystal structure of $\left[S-\left(R^{*}, R^{*}\right)\right]-1-[3-(2,2-$ dimethyl-4-phenyloxazolidine- 3-yl)-1-methyl-2-[methylidene-3-oxopropyl]-4-phenyl-1,2,4-triazolidine- 3,5-dione, $\mathrm{C}_{11} \mathrm{H}_{14} \mathrm{NO}\left(\mathrm{C}_{5} \mathrm{H}_{6} \mathrm{O}\right) \mathrm{C}_{8} \mathrm{H}_{6} \mathrm{~N}_{3} \mathrm{O}_{2}$
}

\author{
K. Peters, E.-M. Peters
}

Max-Planck-Institut für Festkörperforschung, Heisenbergstraße 1, D-70506 Stuttgart, Germany

W. Adam, A. Pastor and Th. Wirth

Universität Würzburg, Institut für Organische Chemie, Am Hubland, D-97074 Würzburg, Germany

Received July 31, 1998, CSD-No. 409354

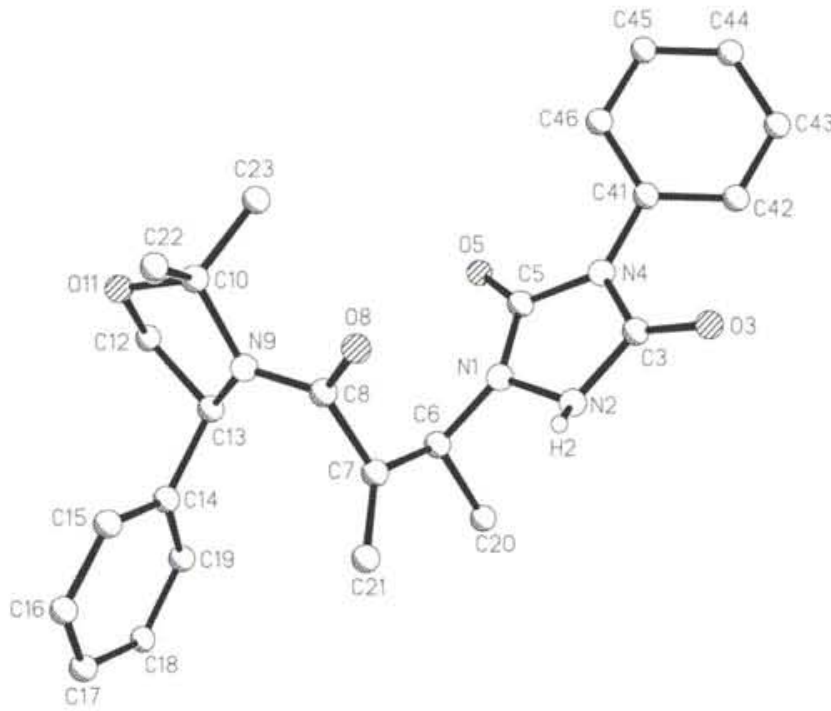

Table 1. Data collection and handling.

$\begin{array}{ll}\text { Crystal: } & \text { colorless prism, size } 0.4 \times 0.45 \times 0.5 \mathrm{~mm} \\ \text { Wavelength: } & \text { Mo } K_{\alpha} \text { radiation }(0.71073 \AA) \\ \mu: & 0.90 \mathrm{~cm}^{-1} \\ \text { Diffractometer, scan mode: } & \text { Siemens P4, } \omega \\ 2 \theta_{\text {max: }} & 55^{\circ} \\ N(h k l)_{\text {measured, }} N(h k l)_{\text {unique: }} & 7042,5361 \\ \text { Criterion for } F_{\text {obs }}, N(h k l)_{\text {grt }}: & F_{\text {obs }}>3 \sigma\left(F_{\text {obs }}\right), 3820 \\ N(\text { param })_{\text {refined: }} & 299 \\ \text { Program: } & \text { SHELXTL-plus [2] }\end{array}$

\begin{abstract}
$\mathrm{C}_{24} \mathrm{H}_{26} \mathrm{~N}_{4} \mathrm{O}_{4}$, orthorhombic, $P 2{ }_{1}{ }_{2}{ }_{2} 1$ (No. 19), $a=9.729$ (1) $\AA$, $b=14.524$ (1) $\AA, c=16.498$ (1) $\AA, V=2331.2 \AA^{3}, Z=4$, $R_{\mathrm{gt}}(F)=0.052, R_{\mathrm{w}}(F)=0.045, T=293 \mathrm{~K}$.
\end{abstract}

\section{Source of material}

The title compound was prepared according to [1].

Table 2. Atomic coordinates and displacement parameters (in $\AA^{2}$ ).

\begin{tabular}{llllll}
\hline Atom & Site & $x$ & $y$ & $z$ & $U_{\text {iso }}$ \\
\hline & & & & & \\
$\mathrm{H}(2)$ & $4 a$ & $0.628(3)$ & $0.721(2)$ & $0.428(2)$ & 0.08 \\
$\mathrm{H}(6)$ & $4 a$ & $0.9084(2)$ & $0.8669(2)$ & $0.4954(1)$ & 0.08 \\
$\mathrm{H}(12 \mathrm{~A})$ & $4 a$ & $0.8763(3)$ & $1.1484(2)$ & $0.6441(2)$ & 0.08 \\
$\mathrm{H}(12 \mathrm{~B})$ & $4 a$ & $0.9027(3)$ & $1.0487(2)$ & $0.6771(2)$ & 0.08 \\
$\mathrm{H}(13)$ & $4 a$ & $0.8942(2)$ & $1.0132(2)$ & $0.5415(1)$ & 0.08 \\
$\mathrm{H}(15)$ & $4 a$ & $0.6121(3)$ & $1.1635(2)$ & $0.5236(2)$ & 0.08 \\
$\mathrm{H}(16)$ & $4 a$ & $0.6000(4)$ & $1.2770(2)$ & $0.4258(2)$ & 0.08 \\
$\mathrm{H}(17)$ & $4 a$ & $0.7865(6)$ & $1.3047(2)$ & $0.3404(2)$ & 0.08 \\
$\mathrm{H}(18)$ & $4 a$ & $0.9872(4)$ & $1.2180(3)$ & $0.3559(2)$ & 0.08 \\
$\mathrm{H}(19)$ & $4 a$ & $0.9969(3)$ & $1.1021(2)$ & $0.4528(2)$ & 0.08 \\
$\mathrm{H}(20 \mathrm{~A})$ & $4 a$ & $0.9609(3)$ & $0.8798(2)$ & $0.3629(2)$ & 0.08 \\
$\mathrm{H}(20 \mathrm{~B})$ & $4 a$ & $0.8687(3)$ & $0.7942(2)$ & $0.3428(2)$ & 0.08 \\
$\mathrm{H}(20 \mathrm{C})$ & $4 a$ & $1.0013(3)$ & $0.7822(2)$ & $0.3958(2)$ & 0.08 \\
$\mathrm{H}(21 \mathrm{~A})$ & $4 a$ & $0.731(3)$ & $0.926(2)$ & $0.331(2)$ & 0.08 \\
$\mathrm{H}(21 \mathrm{~B})$ & $4 a$ & $0.609(3)$ & $0.991(2)$ & $0.381(2)$ & 0.08 \\
$\mathrm{H}(22 \mathrm{~A})$ & $4 a$ & $0.4369(3)$ & $0.9754(3)$ & $0.6457(2)$ & 0.08 \\
$\mathrm{H}(22 \mathrm{~B})$ & $4 a$ & $0.4700(3)$ & $1.0786(3)$ & $0.6258(2)$ & 0.08 \\
$\mathrm{H}(22 \mathrm{C})$ & $4 a$ & $0.4639(3)$ & $1.0455(3)$ & $0.7162(2)$ & 0.08 \\
$\mathrm{H}(23 \mathrm{~A})$ & $4 a$ & $0.6284(4)$ & $0.8705(2)$ & $0.6922(2)$ & 0.08 \\
$\mathrm{H}(23 \mathrm{~B})$ & $4 a$ & $0.6534(4)$ & $0.9359(2)$ & $0.7663(2)$ & 0.08 \\
$\mathrm{H}(23 \mathrm{C})$ & $4 a$ & $0.7747(4)$ & $0.9124(2)$ & $0.7073(2)$ & 0.08 \\
$\mathrm{H}(42)$ & $4 a$ & $0.8157(3)$ & $0.4448(2)$ & $0.5156(2)$ & 0.08 \\
$\mathrm{H}(43)$ & $4 a$ & $0.8518(4)$ & $0.3227(3)$ & $0.6045(3)$ & 0.08 \\
$\mathrm{H}(44)$ & $4 a$ & $0.8674(4)$ & $0.3484(3)$ & $0.7429(3)$ & 0.08 \\
$\mathrm{H}(45)$ & $4 a$ & $0.8464(3)$ & $0.4961(3)$ & $0.7943(2)$ & 0.08 \\
$\mathrm{H}(46)$ & $4 a$ & $0.8054(3)$ & $0.6217(2)$ & $0.7059(2)$ & 0.08 \\
& & & & &
\end{tabular}


Table 3. Atomic coordinates and displacement parameters (in $\AA^{2}$ ).

\begin{tabular}{|c|c|c|c|c|c|c|c|c|c|c|}
\hline Atom & Site & $x$ & $y$ & $z$ & $U_{11}$ & $U_{22}$ & $U_{33}$ & $U_{12}$ & $U_{13}$ & $U_{23}$ \\
\hline $\mathrm{N}(1)$ & $4 a$ & $0.7859(2)$ & $0.7550(1)$ & $0.4933(1)$ & $0.047(1)$ & $0.040(1)$ & $0.050(1)$ & $-0.002(1)$ & $-0.0053(9)$ & $0.0001(9)$ \\
\hline$N(2)$ & $4 a$ & $0.7036(2)$ & $0.6980(1)$ & $0.4445(1)$ & $0.043(1)$ & $0.048(1)$ & $0.059(1)$ & $0.001(1)$ & $-0.010(1)$ & $-0.001(1)$ \\
\hline$C(3)$ & $4 a$ & $0.6939(2)$ & $0.6141(2)$ & $0.4828(1)$ & $0.035(1)$ & $0.050(1)$ & $0.063(1)$ & $0.002(1)$ & $-0.000(1)$ & $-0.003(1)$ \\
\hline$O(3)$ & $4 a$ & $0.6229(2)$ & $0.5502(1)$ & $0.4616(1)$ & $0.051(1)$ & $0.054(1)$ & $0.096(1)$ & $-0.010(1)$ & $-0.011(1)$ & $-0.002(1)$ \\
\hline $\mathrm{N}(4)$ & $4 a$ & $0.7820(2)$ & $0.6186(1)$ & $0.5493(1)$ & $0.039(1)$ & $0.043(1)$ & $0.056(1)$ & $-0.000(1)$ & $0.003(1)$ & $0.003(1)$ \\
\hline$C(5)$ & $4 a$ & $0.8427(2)$ & $0.7061(2)$ & $0.5537(1)$ & $0.041(1)$ & $0.044(1)$ & $0.046(1)$ & $0.005(1)$ & $0.008(1)$ & $-0.005(1)$ \\
\hline $\mathrm{O}(5)$ & $4 a$ & $0.9284(2)$ & $0.7322(1)$ & $0.60262(9)$ & $0.0497(9)$ & $0.060(1)$ & $0.0470(9)$ & $-0.0019(9)$ & $-0.0013(8)$ & $-0.0014(8)$ \\
\hline$C(6)$ & $4 a$ & $0.8425(2)$ & $0.8400(2)$ & $0.4592(1)$ & $0.043(1)$ & $0.042(1)$ & $0.051(1)$ & $-0.002(1)$ & $0.004(1)$ & $0.001(1)$ \\
\hline$C(7)$ & $4 a$ & $0.7253(2)$ & $0.9085(1)$ & $0.4495(1)$ & $0.042(1)$ & $0.039(1)$ & $0.042(1)$ & $-0.004(1)$ & $0.000(1)$ & $-0.001(1)$ \\
\hline $\mathrm{C}(8)$ & $4 a$ & $0.6464(2)$ & $0.9262(2)$ & $0.5266(1)$ & $0.039(1)$ & $0.046(1)$ & $0.044(1)$ & $-0.000(1)$ & $0.000(1)$ & $0.004(1)$ \\
\hline$O(8)$ & $4 a$ & $0.5466(2)$ & $0.8790(1)$ & $0.5439(1)$ & $0.055(1)$ & $0.070(1)$ & $0.0560(9)$ & $-0.021(1)$ & $0.0067(9)$ & $-0.0052(9)$ \\
\hline$N(9)$ & $4 a$ & $0.6938(2)$ & $0.9935(1)$ & $0.57563(9)$ & $0.045(1)$ & $0.041(1)$ & $0.0431(9)$ & $-0.004(1)$ & $0.0022(8)$ & $-0.0009(9)$ \\
\hline$C(10)$ & $4 a$ & $0.6403(3)$ & $1.0060(2)$ & $0.6592(1)$ & $0.074(2)$ & $0.059(2)$ & $0.045(1)$ & $-0.009(2)$ & $0.000(1)$ & $-0.007(1)$ \\
\hline$O(11)$ & $4 a$ & $0.7103(3)$ & $1.0873(1)$ & $0.6840(1)$ & $0.105(2)$ & $0.071(1)$ & $0.057(1)$ & $-0.012(1)$ & $-0.003(1)$ & $-0.0163(9)$ \\
\hline$C(12)$ & $4 a$ & $0.8403(3)$ & $1.0869(2)$ & $0.6471(2)$ & $0.071(2)$ & $0.070(2)$ & $0.073(2)$ & $-0.015(2)$ & $-0.019(2)$ & $0.000(2)$ \\
\hline$C(13)$ & $4 a$ & $0.8184(2)$ & $1.0482(2)$ & $0.5624(1)$ & $0.045(1)$ & $0.041(1)$ & $0.062(1)$ & $-0.003(1)$ & $-0.004(1)$ & $-0.000(1)$ \\
\hline$C(14)$ & $4 a$ & $0.8046(3)$ & $1.1217(2)$ & $0.4977(1)$ & $0.054(1)$ & $0.042(1)$ & $0.057(1)$ & $-0.008(1)$ & $-0.001(1)$ & $-0.006(1)$ \\
\hline$C(15)$ & $4 a$ & $0.6895(3)$ & $1.1740(2)$ & $0.4888(2)$ & $0.068(2)$ & $0.069(2)$ & $0.095(2)$ & $0.008(2)$ & $0.003(2)$ & $0.027(2)$ \\
\hline$C(16)$ & $4 a$ & $0.6823(4)$ & $1.2411(2)$ & $0.4312(2)$ & $0.095(3)$ & $0.082(2)$ & $0.138(3)$ & $0.011(2)$ & $-0.020(3)$ & $0.037(2)$ \\
\hline$C(17)$ & $4 a$ & $0.7919(6)$ & $1.2577(2)$ & $0.3811(2)$ & $0.144(4)$ & $0.078(2)$ & $0.084(2)$ & $-0.037(3)$ & $-0.027(3)$ & $0.029(2)$ \\
\hline$C(18)$ & $4 a$ & $0.9092(4)$ & $1.2064(3)$ & $0.3900(2)$ & $0.111(3)$ & $0.086(3)$ & $0.077(2)$ & $-0.036(2)$ & $0.015(2)$ & $0.012(2)$ \\
\hline$C(19)$ & $4 a$ & $0.9150(3)$ & $1.1385(2)$ & $0.4474(2)$ & $0.072(2)$ & $0.064(2)$ & $0.081(2)$ & $-0.015(2)$ & $0.012(2)$ & $-0.004(2)$ \\
\hline $\mathrm{C}(20)$ & $4 a$ & $0.9260(3)$ & $0.8224(2)$ & $0.3832(2)$ & $0.060(2)$ & $0.069(2)$ & $0.071(2)$ & $0.014(2)$ & $0.016(1)$ & $0.003(2)$ \\
\hline$C(21)$ & $4 a$ & $0.6864(3)$ & $0.9452(2)$ & $0.3811(2)$ & $0.058(2)$ & $0.062(2)$ & $0.051(1)$ & $0.006(2)$ & $0.005(1)$ & $0.003(1)$ \\
\hline $\mathrm{C}(22)$ & $4 a$ & $0.4891(3)$ & $1.0284(3)$ & $0.6620(2)$ & $0.086(2)$ & $0.114(3)$ & $0.079(2)$ & $0.004(2)$ & $0.029(2)$ & $-0.023(2)$ \\
\hline$C(23)$ & $4 a$ & $0.6776(4)$ & $0.9236(2)$ & $0.7110(2)$ & $0.126(3)$ & $0.082(2)$ & $0.057(2)$ & $-0.024(2)$ & $-0.007(2)$ & $0.013(2)$ \\
\hline$C(41)$ & $4 a$ & $0.8073(2)$ & $0.5438(2)$ & $0.6035(2)$ & $0.036(1)$ & $0.056(2)$ & $0.072(2)$ & $-0.002(1)$ & $0.009(1)$ & $0.017(1)$ \\
\hline$C(42)$ & $4 a$ & $0.8212(3)$ & $0.4557(2)$ & $0.5729(2)$ & $0.075(2)$ & $0.052(2)$ & $0.100(2)$ & $0.001(2)$ & $0.000(2)$ & $0.014(2)$ \\
\hline$C(43)$ & $4 a$ & $0.8429(4)$ & $0.3841(3)$ & $0.6253(3)$ & $0.115(3)$ & $0.060(2)$ & $0.159(4)$ & $-0.001(2)$ & $-0.005(3)$ & $0.037(3)$ \\
\hline$C(44)$ & $4 a$ & $0.8518(4)$ & $0.3991(3)$ & $0.7069(3)$ & $0.093(3)$ & $0.106(3)$ & $0.151(4)$ & $-0.010(3)$ & $-0.009(3)$ & $0.081(3)$ \\
\hline $\mathrm{C}(45)$ & $4 a$ & $0.8393(3)$ & $0.4859(3)$ & $0.7370(2)$ & $0.072(2)$ & $0.127(3)$ & $0.088(2)$ & $-0.014(2)$ & $0.008(2)$ & $0.046(3)$ \\
\hline$C(46)$ & $4 a$ & $0.8156(3)$ & $0.5604(2)$ & $0.6849(2)$ & $0.059(2)$ & $0.084(2)$ & $0.067(2)$ & $0.000(2)$ & $0.010(1)$ & $0.018(2)$ \\
\hline
\end{tabular}

\section{References}

1. Adam, W.; Guethlein, M.; Peters, E.-M.; Peters, K.; Wirth, Th.: ChiralAuxiliary-Induced Diastereoselectivity in the $[4+2]$ Cycloadditions of Optically Active 2,2-Dimethyloxazolidine Derivates of Sorbic Acid: A Model Study with Singlet Oxygen as the Smallest Dienophile. J. Am. Chem. Soc. 120 (1998) 4091-4093.

2. Sheldrick, G. M.: Program Package SHELXTL-plus. Release 4.1. Siemens Analytical X-Ray Instruments Inc., Madison (WI 53719), USA 1990. 